\title{
INEQUALITIES FOR SUMS OF INDEPENDENT RANDOM VARIABLES IN LORENTZ SPACES
}

\author{
GHADIR SADEGHI
}

\begin{abstract}
By using interpolation with a function parameter, we establish a moment inequality for sums of independent random variables in Lorentz spaces $\Lambda^{p}(\varphi)$. These estimates generalize Rosenthal inequalities in the LorentzZygmund spaces $L^{p, q}(\log L)^{\gamma}$ as well as Lorentz spaces $L^{p, q}$.
\end{abstract}

1. Introduction. We begin our work by recalling the classical Khintchine inequalities. Let $\left\{r_{k}\right\}_{k \geq 1}$ be a Rademacher sequence on a probability space $(\Omega, \mathfrak{F}, P)$. Since $\left\{r_{k}\right\}_{k \geq 1}$ is an orthogonal sequence in $L^{2}(\Omega)$, for any finite sequence $\left\{\alpha_{k}\right\} \subseteq \mathbf{C}$

$$
\left\|\sum_{k} \alpha_{k} r_{k}\right\|_{2}=\left(\sum_{k}\left|\alpha_{k}\right|^{2}\right)^{1 / 2} \text {. }
$$

The classical Khintchine inequalities assert that $\left\|\sum_{k} \alpha_{k} r_{k}\right\|_{2}$ is uniformly equivalent to $\left\|\sum_{k} \alpha_{k} r_{k}\right\|_{p}$ for any $p<\infty$, namely,

$$
\left\|\sum_{k} \alpha_{k} r_{k}\right\|_{p} \approx\left(\sum_{k}\left|\alpha_{k}\right|^{2}\right)^{1 / 2} .
$$

The equivalence $A \approx B$ means that $c_{1} A \leq B \leq c_{2} A$ for some positive constants $c_{1}$ and $c_{2}$. Rosenthal [12] generalized the Khintchine inequality by replacing $\left\{r_{k}\right\}_{k \geq 1}$ with an arbitrary sequence $\left\{X_{k}\right\}_{k \geq 1}$ of independent symmetric random variables on a probability space $(\Omega, \mathfrak{F}, p)$. More precisely, he proved that, for such a sequence $\left\{X_{k}\right\}_{k \geq 1} \subset L^{p}(\Omega)$, $p>2$, we have

$$
\left\|\sum_{k=1}^{n} X_{k}\right\|_{p} \approx \max \left\{\left\|\sum_{k=1}^{n} X_{k}\right\|_{2},\left(\sum_{k=1}^{n}\left\|X_{k}\right\|_{p}^{p}\right)^{1 / p}\right\}
$$

2010 AMS Mathematics subject classification. Primary 46E30, Secondary $60 \mathrm{G} 50$

Keywords and phrases. Random variable, Rosenthal inequality, Lorentz space, interpolation.

Received by the editors on September 21, 2013.

DOI: 10.1216/RMJ-2015-45-5-1631

Copyright (C)2015 Rocky Mountain Mathematics Consortium 
for all $n \geq 1$. Carothers and Dilworth [3] proved an analogous result for some of the Lorentz spaces, namely, for $2<p<\infty, 0<q \leq \infty$, and any independent symmetric random variables $X_{1}, X_{2}, \ldots, X_{n}$,

$$
\left\|\sum_{k=1}^{n} X_{k}\right\|_{L^{p, q}(\Omega)} \approx \max \left\{\left\|\sum_{k=1}^{n} X_{k}\right\|_{L^{2}(\Omega)},\left\|\sum_{k=1}^{n} \bigoplus X_{k}\right\|_{L^{p, q}(0, \infty)}\right\},
$$

where $\sum_{k=1}^{n} \bigoplus X_{k}$ denotes the disjoint sum of $X_{1}, X_{2}, \ldots, X_{n}$, which is a function on $(0, \infty)$ with $d_{X}(t)=\sum_{k=1}^{n} d_{X_{k}}(t)$. For example, we could take $X(t)=\sum_{i=1}^{n} X_{i}(t-i+1) \chi_{[i-1, i]}$ for $0 \leq t \leq n$. In the setting symmetric function spaces, Johnson and Schechtman [7] established a generalization of Rosenthal inequalities. Recently, $\mathrm{Hu}$ [6] generalize Rosenthal inequalities to $p \geq 0$ instead of $p>2$ and replaced the quantity 2 by $r \in[1,2]$ for conditionally independent mean zero random variables.

In this paper, by use of interpolation with a function parameter, a moment inequality is proved for sums of independent random variables in Lorentz spaces $\Lambda^{q}(\Omega)$. These estimates generalize Rosenthal inequalities in the Lorentz-Zygmund spaces $L^{p, q}(\log L)^{\gamma}$ as well as Lorentz spaces $L^{p, q}$.

2. Lorentz spaces $\Lambda_{\Omega}^{q}(\varphi)$. Let $(\Omega, \Sigma, \mu)$ be a $\sigma$-finite nonatomic measure space. For a given weight $\omega$, let $L_{\mu}^{p}(\omega)$ denote the Lebesgue space defined by the norm $\|f\|_{L_{\mu}^{p}(\omega)}=\|f \omega\|_{L^{p}(\mu)}$ and $L_{*}^{p}(\omega)$ when the measure is $d t / t$ on $\mathbb{R}^{+}=(0, \infty)$.

Definition 2.1. We say that function $f:(0, \infty) \rightarrow(0, \infty)$ belongs to the class $\mathfrak{B}$ if $f(1)=1, f$ is continuous and

$$
\bar{f}(t)=\sup _{s>0} \frac{f(t s)}{f(s)}<\infty,
$$

for all $0<t<\infty$.

For such a function $f$, the Boyd upper and lower indices $\alpha_{\bar{f}}$ and $\beta_{\bar{f}}$ ([10]) of $\bar{f}$, which is submultiplicative and Lebesgue-measurable, are 
then defined by

$$
\alpha_{\bar{f}}=\lim _{t \rightarrow+\infty} \frac{\log \bar{f}(t)}{\log t}, \quad \beta_{\bar{f}}=\lim _{t \rightarrow 0} \frac{\log \bar{f}(t)}{\log t}
$$

with

$$
-\infty<\beta_{\bar{f}} \leq \alpha_{\bar{f}}<+\infty .
$$

For example, if $\theta, \gamma \in \mathbf{R}$, then $f(t)=t^{\theta}(1+|\log t|)^{\gamma} \in \mathfrak{B}, \bar{f}(t)=$ $t^{\theta}(1+|\log t|)^{|\gamma|}$ and $\alpha_{\bar{f}}=\beta_{\bar{f}}=\theta$.

Let $\varphi \in \mathfrak{B}$ and $0<q \leq \infty$; the Lorentz space $\Lambda^{q}(\varphi)$ is the set of (classical of) $\mu$-measurable functions from $\Omega$ in $\mathbf{C}$ such that

$$
\begin{aligned}
\|f\|_{\Lambda_{\Omega}^{q}(\varphi)}:=\left\|f^{*}\right\|_{L_{*}^{q}(\varphi)} & =\left(\int_{0}^{\infty}\left(\varphi(t) f^{*}(t)\right)^{q} \frac{d t}{t}\right)^{1 / q}<\infty \\
(0 & <q<\infty) \\
\|f\|_{\Lambda_{\Omega}^{\infty}(\varphi)}:=\left\|f^{*}\right\|_{L_{*}^{\infty}(\varphi)} & =\sup _{t>0} \varphi(t) f^{*}(t)<\infty,
\end{aligned}
$$

where $f^{*}$ denotes the decreasing rearrangement of $|f|$, i.e.,

$$
f^{*}(t)=\inf \left\{s>0: d_{f}(s)=\mu(\{|f|>s\}) \leq t\right\} .
$$

It is known that $\Lambda_{\Omega}^{q}(\varphi)$ is a rearrangement quasi-Banach space.

Remark 2.2. It is also well known that the inclusion relations between Lorentz spaces are determined by their fundamental functions, since $\Lambda_{\Omega}^{q}\left(\varphi_{1}\right) \subset \Lambda_{\Omega}^{q}\left(\varphi_{2}\right)$ if and only if $\omega_{2}(t) \leq C \omega_{1}(t)$ for all $t>0$, and both spaces agree if and only if $\omega_{1} \approx \omega_{2}$, where

$$
\omega_{i}(t)=\left(\int_{0}^{t} \varphi(s)^{q} \frac{d s}{s}\right)^{1 / q}
$$

is the fundamental function for $\Lambda_{\Omega}^{q}\left(\varphi_{i}\right), i=1,2,[4]$.

Example 2.3. For $\varphi(t)=t^{1 / p}(1+|\log t|)^{\gamma}$ with $0<p<\infty$ and $-\infty<\gamma<+\infty, \Lambda_{\Omega}^{q}(\varphi)$ is the Lorentz-Zygmund space $L^{p, q}(\log L)^{\gamma}$. This is the classical Lorentz space $L^{p, q}$ if $\gamma=0$.

We let $\left(\mathcal{A}_{1}, \mathcal{A}_{2}\right)$ denote a compatible couple of quasi-Banach spaces pair (i.e., $\mathcal{A}_{1}$ and $\mathcal{A}_{2}$ are quasi-Banach spaces, which are continuously 
embedded in some Hausdorff topological vector space) and $K$ is the classical interpolation functional of Peetre.

$$
\begin{gathered}
K(t, a)=K\left(t, a, \mathcal{A}_{1}, \mathcal{A}_{2}\right)=\inf \left\{\left\|a_{1}\right\|_{\mathcal{A}_{1}}+t\left\|a_{2}\right\|_{\mathcal{A}_{2}}: a=a_{1}+a_{2}\right\} \\
t>0 .
\end{gathered}
$$

We can define, for each $p, 0<p \leq \infty$ and each Lebesgue-measurable function $f:(0, \infty) \rightarrow(0, \infty)$, the space

$$
\begin{aligned}
\left(\mathcal{A}_{1}, \mathcal{A}_{2}\right)_{f, p ; K} & =\left\{a: a \in \mathcal{A}_{1}+\mathcal{A}_{2},\|a\|_{f, p ; K}\right. \\
& \left.=\left\|K\left(t, a ; \mathcal{A}_{1}, \mathcal{A}_{2}\right) / f(t)\right\|_{L_{*}^{q}(0, \infty)}<\infty\right\} .
\end{aligned}
$$

The space $\left(\mathcal{A}_{1}, \mathcal{A}_{2}\right)_{f, p ; K}$ is quasi-normed by $\|\cdot\|_{f, p ; K}$. To generalize to $\left(\mathcal{A}_{1}, \mathcal{A}_{2}\right)_{f, p ; K}$ the very well known properties of this space when $f(t)=t^{\theta}$ (i.e., $\left.\left(\mathcal{A}_{1}, \mathcal{A}_{2}\right)_{\theta, p ; K}\right)$, one takes the function $f$ in the class $\mathfrak{B}$. In [10], Merucci showed that interpolation with a function parameter is perfectly suited for identifying interpolation spaces between two quasinormed Lorentz spaces $\Lambda_{\Omega}^{q}(\varphi)$. We refer the reader to $[\mathbf{5}, \mathbf{1 0}, \mathbf{1 1}, \mathbf{1 3}]$ for the theory and bibliography concerning these spaces. Recall also that intersection of two Lorentz spaces $\Lambda_{\Omega}^{q}\left(\varphi_{1}\right)$ and $\Lambda_{\Omega}^{q}\left(\varphi_{2}\right)$ is a quasiBanach space under the quasi-norm $\max \left\{\|\cdot\|_{\Lambda_{\Omega}^{q}\left(\varphi_{1}\right)},\|\cdot\|_{\Lambda_{\Omega}^{q}\left(\varphi_{2}\right)}\right\}$.

3. Main results. In the sequel, we assume that $(\Omega, \mathfrak{F}, P)$ is probability space and establish an extension of Rosenthal inequalities in Lorentz spaces $\Lambda_{\Omega}^{q}(\varphi)$. To prove the main result, we need the following lemma.

Lemma 3.1. Let $0<r<p<\infty, f \in \mathfrak{B}$, and $0<q \leq \infty$. Then

$$
\begin{aligned}
& \left(L^{r}(0, \infty), L^{r}(0, \infty) \cap L^{p}(0, \infty)\right)_{f, q ; K} \\
& \quad=L^{r}(0, \infty) \cap\left(L^{r}(0, \infty), L^{p}(0, \infty)\right)_{f, q ; K} .
\end{aligned}
$$

Proof. By use of Holmsted's formula about interpolation with a function parameter $[\mathbf{1 0}, \mathbf{1 1}]$ the proof of this lemma is similar to $[\mathbf{3}$, Lemma 2.1]. 
Theorem 3.2. Given $1 \leq r \leq 2<p<\infty$ and $0<q \leq \infty$, let $f \in \mathfrak{B}$ with $0<\beta_{\bar{f}} \leq \alpha_{\bar{f}}<1$. Then

$$
\left\|\sum_{k=1}^{n} X_{k}\right\|_{\Lambda_{\Omega}^{q}(\varphi)} \approx \max \left\{\left\|\sum_{k=1}^{n} X_{k}\right\|_{L^{r}(\Omega)},\left\|\sum_{k=1}^{n} \bigoplus X_{k}\right\|_{\Lambda_{(0, \infty)}^{q}(\varphi)}\right\},
$$

for all independent symmetric random variables $X_{1}, X_{2}, \ldots, X_{n}$ in $\Lambda_{\Omega}^{q}(\varphi)$, where

$$
\varphi(t)=\frac{t^{1 / r}}{f\left(t^{1 / r-1 / p}\right)} .
$$

Proof. It follows from [10, Theorem 3] that $\varphi \in \mathfrak{B}$. It is convenient to take $\Omega$ be $[0,1]^{\mathbf{N}}$ with the product measure and denote a typical element of $\Omega$ by the sequence $t=\left(t_{1}, t_{2}, \ldots\right)$. Define a linear operator $T: L_{0}(0, \infty) \rightarrow L_{0}(\Omega \times[0,1])$ by

$$
T(g)=\sum_{k=1}^{\infty} g_{k}\left(t_{k}\right) r_{k}(s)
$$

where $g_{k}\left(t_{k}\right)=g\left(t_{k}+k-1\right)$ and $r_{k}(s)$ is the $k$ th Rademacher function. Then, by Hu's inequality [6], $T$ is a bounded operator from $L^{r}(0, \infty) \cap$ $L^{p}(0, \infty)$ into $L^{p}(\Omega \times[0,1])$ for $p>2$. So, by Lemma 3.1 and the interpolation theorem with a function parameter $([\mathbf{1 0}$, Theorem 3$]$ and [5]), $T$ is bounded from $L^{r}(0, \infty) \cap \Lambda_{(0, \infty)}^{q}(\varphi)$ into $\Lambda_{\Omega}^{q}(\varphi)$, where

$$
\varphi(t)=\frac{t^{1 / r}}{f\left(t^{1 / r-1 / p}\right)} .
$$

Therefore, there exists a positive constant $C$ such that

$$
\left\|\sum_{k=1}^{n} X_{k}\right\|_{\Lambda_{\Omega}^{q}(\varphi)} \leq C \max \left\{\left\|\sum_{k=1}^{n} X_{k}\right\|_{L^{r}(\Omega)},\left\|\sum_{k=1}^{n} \bigoplus X_{k}\right\|_{\Lambda_{(0, \infty)}^{q}(\varphi)}\right\} .
$$

It follows from Remark 2.2 that

$$
\left\|\sum_{k=1}^{n} X_{k}\right\|_{L^{2}(\Omega)} \leq C_{1}\left\|\sum_{k=1}^{n} X_{k}\right\|_{\Lambda_{(\Omega)}^{q}(\varphi)}
$$

for a positive constant $C_{1}$. 
Since $1 \leq r \leq 2<p$ and $\alpha_{\bar{f}}<1$, it follows from [10, Propositions $2,3]$ that

$$
\alpha_{\bar{f}\left(t^{1 / r-1 / p}\right)}=\left(\frac{1}{r}-\frac{1}{p}\right) \alpha_{\bar{f}},
$$

and so $\alpha_{\bar{\varphi}}<1$. On the other hand, $\bar{\alpha}_{\Lambda_{\Omega}^{q}(\varphi)}=\alpha_{\bar{\varphi}}<1$, where $\bar{\alpha}_{\Lambda_{\Omega}^{q}(\varphi)}$ are Boyd indices of $\Lambda_{\Omega}^{q}(\varphi)$, [13]. Now, by [8, Theorem 5.8], $\Lambda_{\Omega}^{q}(\varphi)$ has the Kalton property (that is, for

$$
\varphi(t)=\frac{t^{1 / r}}{f\left(t^{1 / r-1 / p}\right)},
$$

$\Lambda_{\Omega}^{q}(\varphi)$ satisfies $\|X\| \leq C\|Y\|$ whenever $X^{* *} \leq Y^{* *}$ (recall that $\left.X^{* *}(t)=t^{-1} \int_{0}^{t} X^{*}(s) d s\right)$ ).

By the definition of the disjoint sum, it is easy to check that

$$
\left(\sum_{k=1}^{n} \bigoplus X_{k}\right)^{* *} \leq\left(\left(\sum_{k=1}^{n}\left|X_{k}\right|^{2}\right)^{1 / 2}\right)^{* *}
$$

Now, by the Kalton property, we have

$$
\left\|\sum_{k=1}^{n} \bigoplus X_{k}\right\|_{L^{q}((0, \infty))} \leq C_{2}\left\|\left(\sum_{k=1}^{n}\left|X_{k}\right|^{2}\right)^{1 / 2}\right\|_{\Lambda_{\Omega}^{q}(\varphi)}
$$

for some positive constant $C_{2}$. Since $\sum_{k=1}^{n} X_{k}$ has the same distribution as $\sum_{k=1}^{n} X_{k}(t) r_{k}(t)$, by the Maurey-Khintchine inequality [9, Theorem 1.d.6] and inequality (3.3), we obtain

$$
\left\|\sum_{k=1}^{n} \bigoplus X_{k}\right\|_{\Lambda_{(0, \infty)}^{q}(\varphi)} \leq C_{3}\left\|\sum_{k=1}^{n} X_{k}\right\|_{\Lambda_{\Omega}^{q}(\varphi)},
$$

for some positive constant $C_{3}$. Therefore, by inequalities (3.2) and (3.4), we get

$$
C^{\prime} \max \left\{\left\|\sum_{k=1}^{n} X_{k}\right\|_{L^{2}(\Omega)},\left\|\sum_{k=1}^{n} \bigoplus X_{k}\right\|_{\Lambda_{(0, \infty)}^{q}(\varphi)}\right\} \leq\left\|\sum_{k=1}^{n} X_{k}\right\|_{\Lambda_{\Omega}^{q}(\varphi)},
$$

where $C^{\prime}=1 / \max \left\{C_{1}, C_{3}\right\}$. So, the desired inequality now follows 
easily since $1 \leq r \leq 2$, i.e.,

$$
C^{\prime} \max \left\{\left\|\sum_{k=1}^{n} X_{k}\right\|_{L^{r}(\Omega)},\left\|\sum_{k=1}^{n} \bigoplus X_{k}\right\|_{\Lambda_{(0, \infty)}^{q}(\varphi)}\right\} \leq\left\|\sum_{k=1}^{n} X_{k}\right\|_{\Lambda_{\Omega}^{q}(\varphi)} .
$$

Thus, inequalities (3.1) and (3.5) imply that

$$
\left\|\sum_{k=1}^{n} X_{k}\right\|_{\Lambda_{\Omega}^{q}(\varphi)} \approx \max \left\{\left\|\sum_{k=1}^{n} X_{k}\right\|_{L^{r}(\Omega)},\left\|\sum_{k=1}^{n} \bigoplus X_{k}\right\|_{\Lambda_{(0, \infty)}^{q}(\varphi)}\right\} .
$$

Corollary 3.3. Given $1 \leq r \leq 2<p<\infty$ and $0<q \leq \infty$, we then have

$\left\|\sum_{k=1}^{n} X_{k}\right\|_{L^{p, q}(\log L)^{\gamma}} \approx \max \left\{\left\|\sum_{k=1}^{n} X_{k}\right\|_{L^{r}(\Omega)},\left\|\sum_{k=1}^{n} \bigoplus X_{k}\right\|_{L^{p, q}(\log L)^{\gamma}}\right\}$, for all independent symmetric random variables $X_{1}, X_{2}, \ldots, X_{n}$ in $L^{p, q}(\log L)^{\gamma}$.

Proof. It is sufficient to consider

$$
f(t)=t^{\theta}\left(1+\frac{p r}{p-r}|\log t|\right)^{-|\gamma|}
$$

in Theorem 3.2.

Remark 3.4. In the previous corollary, if $\gamma=0$ and $r=2(p=q)$, then this corollary implies Rosenthal inequalities (1.2) in Lorentz spaces $L^{p, q}\left(\operatorname{spaces} L^{p}\right)$.

\section{REFERENCES}

1. C. Bennet and R. Sharpley, Interpoloation of operators, Academic Press, Boston, 1988.

2. C. Bergh and J. Löfström, Interpolation spaces, Springer, Berlin, 1976.

3. N.L. Carothers and S.J. Dilworth, Inequalities for sums of independent random variables, Proc. Amer. Math. Soc. 104 (1988), 221-226.

4. M.J. Carro and J. Soria, Weighted Lorentz spaces and the Hardy operator, J. Funct. Anal. 112 (1993), 480-494.

5. J. Gustavsson, A function parameter in connection with interpolation of Banach spaces, Math. Scand. 42 (1978), 286-305. 
6. X.L. Hu, An extension of Rosenthal's inequality, Appl. Math. Comp. 218 (2011), 4638-4640.

7. W.B. Johnson and G. Schehtman, Sums of independent random variables in rearrangement invariant function spaces, Ann. Probab. 17 (1988), 789-808.

8. N.J. Kalton, Compact and strictly singular operators on certain function spaces, Arch. Math. 43 (1986), 66-78.

9. J. Lidenstrauss and L. Tzafriri, Classical Banach spaces II, Function spaces, Springer-Verlag, Berlin, 1979.

10. C. Merucci, Applications of interpolation with a function parameter to Lorentz, Sobolev and Besov spaces, Lect. Notes Math. 1070 (1984), 183-201.

11. L.E. Persson, Interpolation with a parameter function, Math. Scand. 59 (1986), 199-228.

12. H.P. Rosenthal, On the subspaces of $L_{p}(p>2)$ spanned by sequences of independent random variables, Israel J. Math. 8 (1970), 273-303.

13. R. Sharpley, Spaces $\Lambda_{\alpha}(X)$ and interpolation, J. Funct. Anal. 11 (1972), $479-513$.

Department of Mathematics and Computer Sciences, Hakim Sabzevari UniVERSity, P.O. Box 397, SABZEVAR, Iran

Email address: ghadir54@gmail.com, g.sadeghi@hsu.ac.ir 